\title{
Congenital hypertension due to unilateral renal vein thrombosis
}

\author{
D J EVANS, M SILVERMAN, AND N B BOWLEY
}

Department of Histopathology, Institute of Paediatrics and Child Care, and Department of Radiology, Royal Postgraduate Medical School, Hammersmith Hospital, London

SUMMARY A boy was found on the day of birth to have hypertension and radiological evidence of calcification of the left renal vein. Persistent hypokalaemia and hyper-reninaemia in the presence of a small left kidney and normal right kidney led to the decision to perform a left nephrectomy. The biopsied specimen showed old calcified renal vein thrombosis with accompanying medullary necrosis. Postoperatively hypertension resolved.

Neonatal hypertension is rare and in most of the reported cases it has been attributed to thrombosis of a main renal artery or its branches, or to renal artery stenosis; in some cases the cause is unknown. ${ }^{1}$ The patient here reported appears to be the first in whom congenital hypertension followed intrauterine renal vein thrombosis.

\section{Case report}

A boy was born at 34 weeks' of gestation by spontaneous vertex vaginal delivery, 6 days after the onset of acute hydramnios. The membranes had ruptured spontaneously 8 hours before delivery releasing an apparently normal quantity of amniotic fluid. The pregnancy and the mother's previous two pregnancies had been normal.

Although no fetal distress was noted during labour, the infant was born severely asphyxiated (Apgar score 0 ). An adequate circulation was rapidly restored by endotracheal intubation, positive pressure ventilation, and cardiac massage, but regular respiration was not established for 30 minutes. Thereafter, he had a period of asymptomatic hypoglycaemia for several hours, corrected by intravenous $10 \%$ dextrose. At 2 days there was some abdominal distention together with bloody stools, for which total intravenous feeding was given for 6 days by peripheral drip. The abdominal $x$-ray films at that time showed no abnormality except for calcification near the renal pelvis.

The infant initially weighed $2125 \mathrm{~g}$ and had generalised pitting oedema. There was no evidence of heart failure, and the total serum protein level was $45 \mathrm{~g} / \mathrm{l}$. The urine was free of protein, blood, and casts. During the next 3 days he passed copious quantities of dilute urine (specific gravity 1000-1002), and his weight on day 4 was $1680 \mathrm{~g}$ (below the 10th centile for 34 weeks' gestation). During this time, his haemoglobin concentration rose from 17.5 to $21.8 \mathrm{~g} / \mathrm{dl}$, but his plasma urea and electrolytes remained normal.

Hypertension was found on the first day of life, the systolic pressure (Doppler method) remaining between 150 and $175 \mathrm{mmHg}$ in arms and legs during the next 4 weeks. A chest $x$-ray film showed dextraposition of the heart but this disappeared after successful treatment of the hypertension.

Investigations into the cause of hypertension showed normal values for plasma and urinary sodium, urea, and creatinine, and for urinary vanillylmandelic acid, and plasma 17-hydroxyprogesterone. His serum potassium was raised after the diuretic phase. The mean value $( \pm 1 \mathrm{SD})$ in 13 estimations of serum potassium before treatment with drugs was $5.42( \pm 0.55) \mathrm{mmol} / \mathrm{l}$. The plasma renin activity on two occasions was 13700 and $15000 \mathrm{ng} \mathrm{A1}$ per hour per $\mathrm{ml}$ (range in normal newborn infants 208-9100 ng A1 per hour per $\mathrm{ml}$ ).

A plain abdominal $x$-ray film taken on the day of birth showed the presence of abnormal calcification in the region of the left renal pelvis, and this was confirmed in three subsequent $x$-ray films. The left renal abnormality was further shown by a DTPA renal scan which showed little function on the left, and by an intravenous pyelogram which showed a tiny left kidney; an ultrasound scan suggested that the left kidney was not cystic. The right kidney was large, functioned well, and had normal calyces.

It was felt that the unilateral renal anomaly was the cause of this infant's hypertension. In view of this, and after an episode of cardiac failure had been treated with digoxin, frusemide, and propranolol, a left nephrectomy was performed 7 weeks after birth. It was not possible to measure the left renal vein renin level because of difficulty in sampling from the vein. The infant's subsequent course was uncomplicated. The mild residual hypertension resolved during the next 2 months, requiring no treatment. Four subsequent values of plasma renin activity were normal. At 18 months of age the child is normotensive, and normal in size and psychomotor development. 


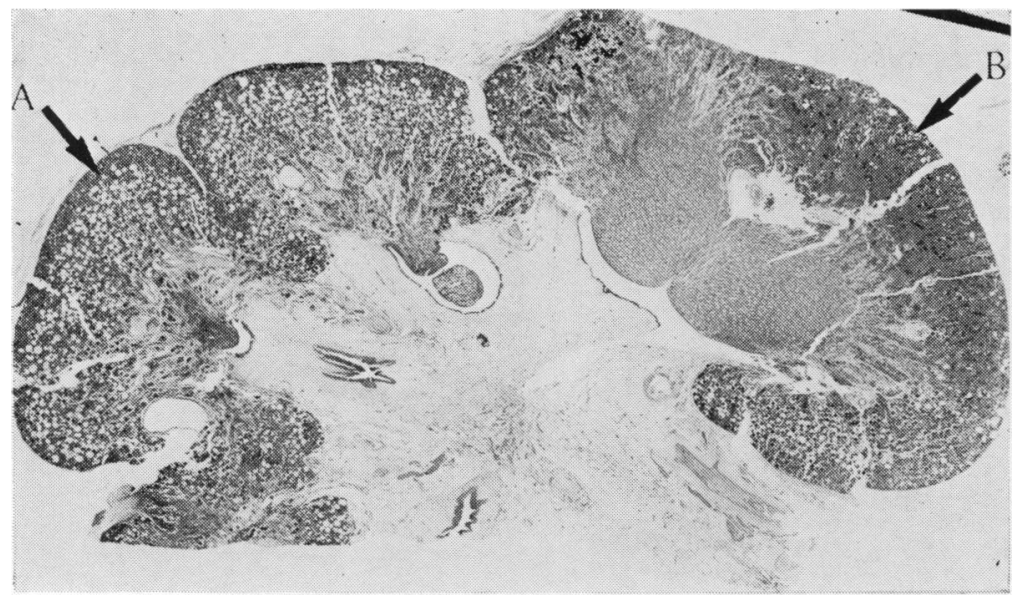

Fig. 1 Whole mount of section of kidney. There are abnormal pyramids with an overlying cortex containing microcysts $(A)$. $A$ normal pyramid and overlying cortex are also shown $(B)$. ( $H$ and $E \times 6 \cdot 7)$.

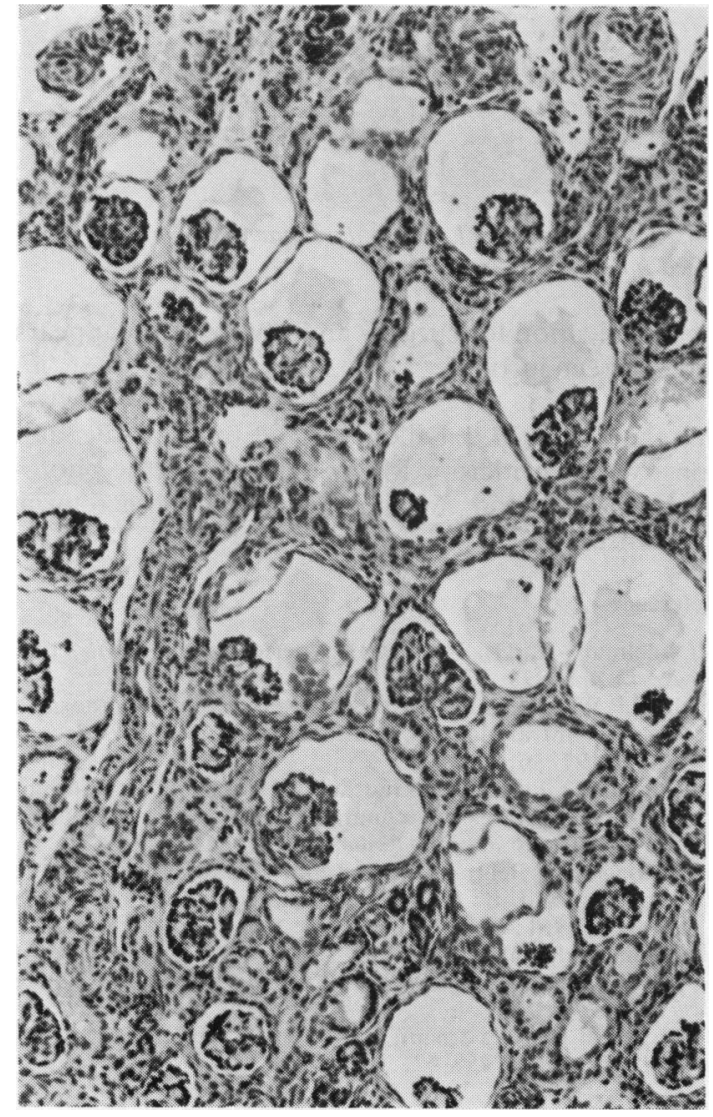

Fig. 2 Many of the glomeruli have dilated spaces around them. Tubules are grossly atrophic. $(H$ and $E \times 150)$.
Pathology. The excised left kidney measured $3 \times 2 \times 1.5 \mathrm{~cm}$. On cut surface almost all the pyramids were abnormal as was the overlying cortex (Figs 1 and 2). Two of the pyramids appeared to be normal and the cortex associated with them was well developed.

The abnormal pyramids showed partial preservation of their architecture at the tips but most of the medulla was disorganised, with loss of tubules, which were replaced by primitive connective tissue interspersed with haemosiderin-laden macrophages.

The overlying cortex showed a marked shrinkage, with disappearance of normal proximal convoluted tubes. The glomeruli (except in the most superficial region of cortex) showed dilatation of Bowman's capsule. The cortical tubules had scanty cytoplasm.

The renal vein and many of its tributaries were distended by old organised and calcified thrombus (Fig. 3). The lumina of renal artery branches were narrowed by fibrointimal thickening but there was no evidence of primary stenosis or thrombotic occlusions of these vessels. The changes were interpreted as renal medullary necrosis associated with renal vein thrombosis.

\section{Discussion}

There seems little doubt that the patient sustained damage to the left kidney in utero, probably a few days before the acute hydramnios. In the neonatal period renal vein thrombosis and medullary necrosis may be followed by a period of salt and water loss, and "similar sequence of events in utero may have produced the hydramnios. The $x$-ray films of the abdomen confirmed the presence of left-sided renal vein calcification at birth. Calcification may supervene upon renal vein thrombosis in the neonatal period, ${ }^{2-4}$ within a few days, and its presence cannot 


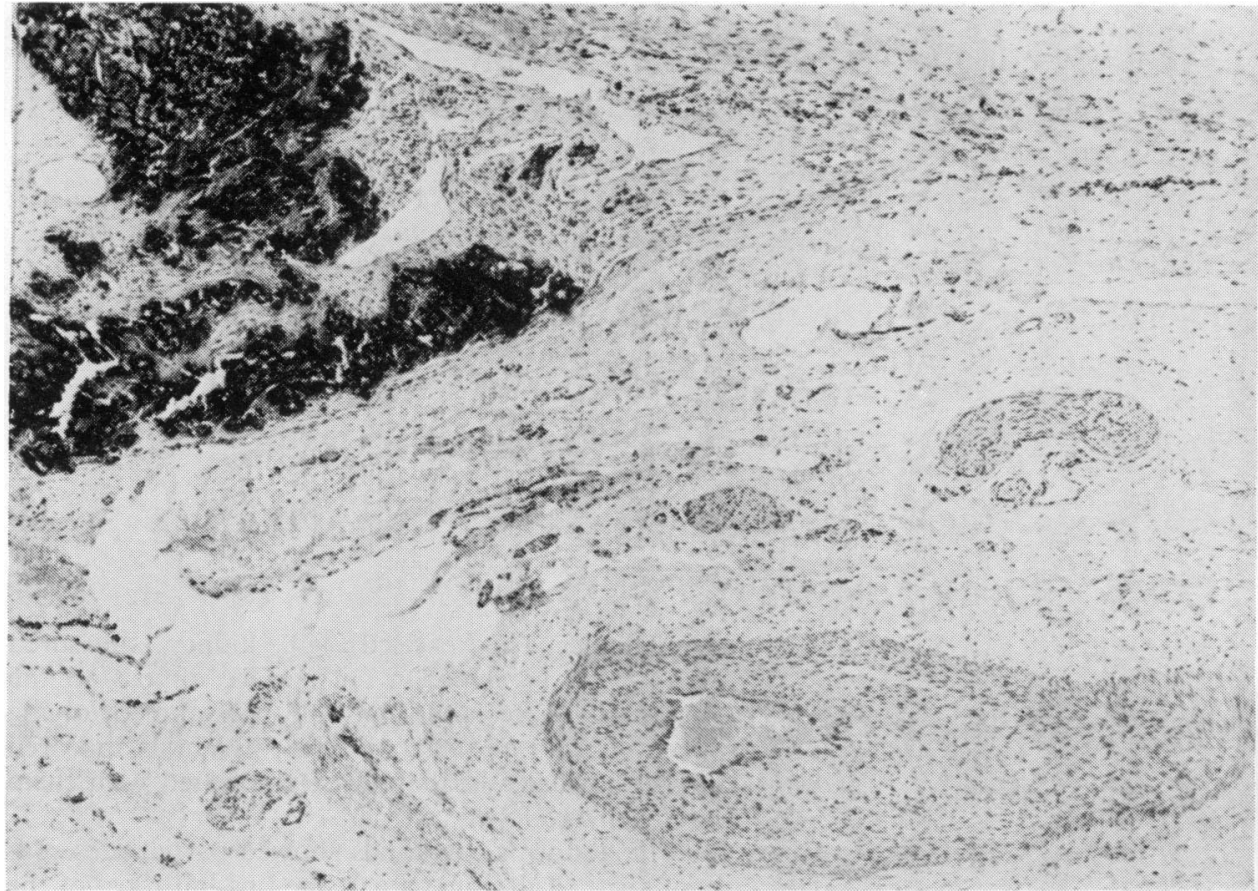

Fig. 3 The renal vein (above left) is greatly distended by organised thrombus which shows dark haematoxyphil calcification. The accompanying artery (below right) shows marked intimal thickening.

be used to date the thrombosis. In view of the radiological appearances of the right kidney it would appear that the pyramids on that side were normal.

Renal medullary necrosis and renal vein thrombosis may be associated, or each may occur independently. ${ }^{5}$ In this instance the involvement of a single kidney and the evidence of old haemorrhage strongly suggest that the primary lession was the venous thrombosis.

In the neonate, hypertension is a common sequel both of renal vein thrombosis and medullary necrosis, though rarely a feature of the acute illness. In this case there was clear evidence that hypersecretion of renin associated with renal ischaemia was responsible for the hypertension that followed renal vein thrombosis and medullary necrosis. The precise mechanism of the cystic dilatation of Bowman's capsule remains unknown: cysts of the renal corpuscle were discussed by Baxter. ${ }^{6}$ Her series included 2 patients who may have been similar to the present one, in that they had small cysts of the renal corpuscle, small kidneys, and abnormalities of the renal medulla.

Although it has been suggested that neonatal hypertension can be successfully managed medically, ${ }^{1}$ in our patient the existence of a unilateral small ischaemic kidney with hyper-reninaemia seemed a clear indication for surgery and this decision appears to have been justified.

We thank Dr $\mathbf{M} \mathbf{J}$ Dillon for the renin assays and constructive criticism, $\mathrm{Mr} \mathrm{W}$ Hinkes for the photomicrographs, and Mrs P Weller for secretarial assistance.

\section{References}

1 Adelman R D. Neonatal hypertension. Pediatr Clin North Am 1978; 25: 99-110.

2 Brill P W, Mitty H A, Strauss L. Renal vein thrombosis: a cause of intrarenal calcification in the newborn. Pediatr Radiol 1977; 6: 172-5.

3 Sutton T J, Leblanc A, Gauthier N, Hassan M. Radiological manifestations of neonatal renal vein thrombosis on follow-up examination. Radiology 1977 ; 122 : 435-8.

4 Takeuchi A, Benirschke K. Renal vein thrombosis of the newborn and its relation to maternal diabetes. Biol Neonate 1961 ; 3: 237-56.

5 Anand S K, Northway J D, Smith J A. Neonatal renal papillary and cortical necrosis. Am J Dis Child 1977; 131 : 773-7.

6 Baxter T J. Cysts arising in the renal corpuscle. Arch Dis Child 1965; 40: 455-63.

Correspondence to Prof. D J Evans, Department of Histopathology, Royal Postgraduate Medical School, Hammersmith Hospital, Ducane Road, London W12 0HS.

Received 25 March 1980 